\title{
Upregulation of aryl hydrocarbon receptor nuclear translocator 2 in the hippocampi of post-stroke depression rats
}

\author{
CHEN-DI PEI ${ }^{1,2^{*}}$, LU-LU ZHANG ${ }^{1,2 *}$, PENG-GE FEI ${ }^{1,2}$, SHI-NA GU ${ }^{3}$, WEN-QIANG LI ${ }^{2,4}$, JUN-LIN MU $^{2,4}$, \\ XU-SHENG WANG ${ }^{1}$, WEN-SHENG WANG ${ }^{5,6}$, XIANG-YANG ZHANG $^{7}$, ZHAO-HUI ZHANG $^{4}$ and JING-GUI SONG ${ }^{5}$ \\ ${ }^{1}$ Department of Neurology, The Second Affiliated Hospital of Xinxiang Medical University; \\ ${ }^{2}$ Henan Key Laboratory of Biological Psychiatry, Xinxiang Medical University, Xinxiang, Henan 453003; \\ ${ }^{3}$ Department of General Medicine, The First Affiliated Hospital of Xinxiang Medical University, Weihui, Henan 453100; \\ ${ }^{4}$ Department of Psychosomatic Medicine, The Second Affiliated Hospital of Xinxiang Medical University, Xinxiang, \\ Henan 453002; ${ }^{5}$ Department of Neurology, The First Affiliated Hospital of Xinxiang Medical University, \\ Weihui, Henan 453100; ${ }^{6}$ Tuberculosis Research Institute of Xinxiang Medical University, \\ Weihui, Henan 453002, P.R. China; ${ }^{7}$ Department of Psychiatry and Behavioral Sciences, \\ The University of Texas Health Science Center at Houston, Houston, TX 77030, USA
}

Received May 3, 2019; Accepted June 11, 2019

DOI: $10.3892 /$ br.2019.1225

\begin{abstract}
Aryl hydrocarbon receptor nuclear translocator protein 2 (ARNT2), a member of the basic helix-loop-helix superfamily of transcription factors, may serve a vital role in neuronal survival and cell proliferation via formation of heterodimers with hypoxia-inducible factor- $1 \alpha$. Previous studies indicated that ARNT2 levels were elevated in the brains of ischemic rats; however, the involvement of ARNT2 in post-stroke depression (PSD) rats is not well understood. Therefore, the present study aimed to investigate the levels of ARNT2 in the hippocampi of PSD rats, and to clarify the potential association between ARNT2 and behavioral performance. A PSD rat model was established by middle cerebral artery occlusion (MCAO) followed by a 4-week chronic unpredictable mild stress (CUMS) regimen. A sucrose preference test and open field test (OFT) were conducted, and body weight was measured. In addition, reverse transcription-polymerase chain reaction and immunohistochemistry were performed
\end{abstract}

Correspondence to: Dr Jing-Gui Song, Department of Neurology, The First Affiliated Hospital of Xinxiang Medical University, 88 Health Road, Weihui, Henan 453100, P.R. China E-mail: songjg62@126.com

Dr Zhao-Hui Zhang, Department of Psychosomatic Medicine, The Second Affiliated Hospital of Xinxiang Medical University, 388 Jianshe Road, Xinxiang, Henan 453002, P.R. China E-mail: zzhui816@126.com

*Contributed equally

Key words: aryl hydrocarbon receptor nuclear translocator protein 2, post-stroke depression, cognitive impairment, hypoxia-inducible factor 1 , hippocampal regeneration to measure ARNT expression. Results indicated that $\mathrm{MCAO}+\mathrm{CUMS}$ rats had lower weight gain, consumed less sucrose and moved less compared with controls. Furthermore, the mRNA and protein levels of ARNT in MCAO+CUMS rats were increased compared with in controls. The sucrose preference index and horizontal movement distance in the OFT were positively correlated with ARNT mRNA level. Thus, from these findings it was suggested that ARNT2 may be positively associated with improvement of cognitive impairment, and therefore may be a potential target in PSD treatment.

\section{Introduction}

Stroke is among the most common causes of mortality and disability worldwide (1), and is defined as loss of blood supply to the brain by thrombotic, embolic or hemorrhagic events (2). The main consequences include local hypoxia, mitochondria dysfunction and increases in intracellular calcium, all of which lead to neuronal death and subsequent tissue damage due to oxygen and glucose deprivation (3). According to a report by the American Heart Association in 2015, up to 85\% of strokes are ischemic, and $12 \%$ are hemorrhagic (4).

Recently, neuropsychiatric disorders resulting from cerebrovascular diseases have gained increasing attention. An epidemiological study across a diverse population suggested that post-stroke depression (PSD) was among the common complications of stroke, with an incidence rate of more than $30 \%$ within 5 years of stroke occurrence (5). In addition, PSD has been reported to be accompanied by sleep disorders, cognitive impairments, and may also be a predictor of poor recovery, high mortality and morbidity, and a cause of considerable health care costs (6-8). The underlying mechanism of PSD is not clearly understood, although several studies have suggested that it is associated with altered neurotrophic signaling, dysregulation of the hypothalamus-pituitary-adrenal axis, neuroinflammation and biological amine neurotransmitter disorders $(1,8,9)$. 
Drutel et al (10) reported that aryl hydrocarbon receptor (AHR) nuclear translocator protein 2 (ARNT2) may serve a vital role in neuron survival and cell proliferation by forming heterodimers with hypoxia-inducible factor (HIF)-1 $\alpha$, a factor involved in adaptation to hypoxia.

ARNT2 is established as a member of the basic helix-loop-helix (bHLH) superfamily of transcription factors, and shares an amino acid sequence of almost $90 \%$ similarity with ARNT (11). It is primarily expressed in the central nervous system and developing kidney (12), forming a dimer with other basic helix-loop-helix Per-Arnt-Sim homology (bHLH-PAS) transcription factors including AHR and HIF-1 $\alpha$ when responding to xenobiotics and hypoxia, respectively (13). It has been suggested that under hypoxic conditions, HIF-1 $\alpha$ alternatively binds with ARNT or ARNT2, also designated as HIF-1 $\beta$ and HIF-2 $\beta$, respectively, and subsequently activates the hypoxic response element, leading to the expression of target genes involved in glycolysis, erythropoiesis and angiogenesis $(14,15)$. Interestingly, a study involving a population from a high altitude area of Ethiopia demonstrated an association between ARNT2 and hemoglobin levels (16), which aided to further clarify the key role of this protein in the hypoxia response in humans.

Magnetic resonance images have shown that hippocampal volume is reduced in patients with depression $(17,18)$. Depression following stroke events is associated with cognitive dysfunction, which may be indirectly associated with the damage to the hippocampus (19-21). In hypoxic environments, neurons, particularly CA1 pyramidal neurons, are susceptible and vulnerable to post-ischemia cell death (22). It has also been reported that the hippocampal CA3 region regulates learning and memory abilities, particularly the normal operations of learning and memory processes (23). Although Valen et al (24) reported that ARNT2 was highly and preferentially expressed in the hippocampus, and previous research indicated that ARNT2 was also potently expressed in ischemic brain tissue in rats (16), less is known about its expression levels in PSD rats. Therefore, the present study aimed to investigate the levels of ARNT2 in the hippocampus of PSD rats, as well as the potential association between ARNT2 levels and cognitive behavioral function.

\section{Materials and methods}

Animals and groups. Male Sprague-Dawley rats $(\mathrm{n}=32)$ aged 8 weeks, weighing $160 \pm 20 \mathrm{~g}$, were purchased from the Experimental Animal Center of Zhengzhou University, Zhengzhou, China. Rats were housed at $22 \pm 2^{\circ} \mathrm{C}$ with $57 \pm 2 \%$ relative humidity under a 12-h light/dark cycle, and were given food and water ad libitum except during specific times of the experiment. All experiments were conducted in accordance with the National Research Council: Guide for the Care and Use of Laboratory Animals (8th edition), and the rat experimental procedures were approved by the Laboratory Animal Management Commission of the Henan Key Laboratory of Biological Psychiatry, Xinxiang, China.

Rats were allowed to adapt to the environment for 1 week before baseline body weight (BW) and sucrose consumption were measured, and open field tests (OFTs) were performed to ensure the homogeneity of rats. Rats were then randomly divided into four groups ( $\mathrm{n}=8 /$ group): A control group; a chronic unpredictable mild stress (CUMS) group; a middle cerebral artery occlusion (MCAO) group; and an $\mathrm{MCAO}+\mathrm{CUMS}$ group. All rats in the MCAO and MCAO+CUMS groups underwent MCAO surgery, while the rats in the CUMS group were treated with a 4-week CUMS regiment. Finally, rats in the MCAO+CUMS group were exposed to the same 4-week CUMS regiment following surgery.

$M C A O$. Rats were fasted overnight prior to the day of the experiment but were allowed free access to tap water. MCAO was conducted as previously described (25). Briefly, anesthesia was induced with $10 \%$ chloral hydrate [350 mg/kg BW, intraperitoneally (i.p.); Dalian Meilun Biological Technology Co., Ltd., Dalian, China] and a ventral side incision was made to expose the left carotid bifurcation, the left common carotid artery (CCA), the left internal carotid artery (ICA) and the left external carotid artery (ECA). The left ECA and proximal CCA were ligated with a 4-0 silk suture, while the left ICA was occluded by a microvascular clip; a small incision was made in the CCA proximal from the left carotid bifurcation. The microvascular clip was then removed and a nylon thread was inserted through the left CCA and left ECA to occlude the middle cerebral artery. Rats in the control and CUMS groups underwent the same surgery as above excluding threading of the nylon through the left CCA and left ECA. Following surgery, rats were selected according to Longa score, as previously described (25).

CUMS procedure. Following recovery for 1 week, rats in the CUMS groups that underwent surgery were subjected to a CUMS procedure adapted from Willner (26) with minor modifications. A total of seven different stressors were used: Water/food deprivation for $24 \mathrm{~h}$; behavioral restriction for $2 \mathrm{~h}$; wet cage for $24 \mathrm{~h}$; electric foot shocks (average $60 \mathrm{~V}$, $10 \mathrm{sec}$ duration, average 1 shock $/ 5 \mathrm{sec}$ ); forced ice water swimming $\left(4^{\circ} \mathrm{C}, 5-15 \mathrm{~min}\right)$; and tail pinch for $1-2 \mathrm{~min}(27,28)$. Rats in the CUMS and MCAO+CUMS groups were housed in separate cages. Stressors were individually administered and separated from one another by a 1-2 day period over a total of 28 days to account for adaptation of rats to the CUMS regimen.

Behavioral tests $B W$. The $\mathrm{BW}$ of rats was measured prior to surgery and on days 1, 7 and 28 thereafter, and was obtained only during normal eating regiments (29).

Sucrose preference test. The animals were allowed to consume water and $1 \%$ sucrose solution for $12 \mathrm{~h}$ following $24 \mathrm{~h}$ food and water deprivation on the day prior to surgery and on days 7 and 28 thereafter. All other components of the test were performed as previously described $(21,25)$.

OFT. The device for this test consisted of an open-field response box $(100 \times 100 \times 50 \mathrm{~cm}$ with opaque black metal walls; RWD Life Science, Shenzhen, China) and an automatic data acquisition and processing system (Panlab Smart version 2.5.16; Panlab, Barcelona, Spain). Behavior, in terms 
of horizontal and vertical movements, was determined in the open-field response box over $5 \mathrm{~min}$ to measure spontaneous activity, independent ability to explore and cognitive function (30).

Immunohistochemistry. Rats were anesthetized with $10 \%$ chloral hydrate $(350 \mathrm{mg} / \mathrm{kg}$, i.p.) and perfused with $0.9 \%$ saline followed by $4 \%$ paraformaldehyde. The samples of 4 rats per group were used for immunohistochemistry. Brains were removed and dehydrated, after which brain tissue was embedded in Tissue-Tek ${ }^{\circledR}$ O.C.T. Compound (Sakura Finetek USA, Inc., Torrance, CA, USA). Frozen tissue was then sectioned at $15-\mu \mathrm{m}$ thickness on a microtome (Leica CM1850; Leica Microsystems GmbH, Wetzlar, Germany), and immunohistochemistry was performed using a rabbit SP-9001 detection kit (OriGene Technologies, Inc., Beijing, China) according to the manufacturer's instructions. Briefly, sections were blocked with $3 \% \mathrm{H}_{2} \mathrm{O}_{2}$ in deionized water at room temperature for endogenous peroxidase ablation, rinsed in phoshphate buffered saline (PBS) three times and blocked in blocking buffer (normal goat serum for 10-15 min at room temperature). Following removal of blocking buffer, sections were incubated with primary antibody in PBS for $1 \mathrm{~h}$ at $37^{\circ} \mathrm{C}$, washed three times with PBS, and incubated with biotinylated secondary antibody (immunoglobulin G, anti-rabbit) for 10-15 min at room temperature. Sections were then incubated with horseradish enzyme-labeled streptavidin for a further 10-15 min at $37^{\circ} \mathrm{C}$. ARNT-positive staining in the CA1 and CA2 hippocampal fields was observed under an optical microscope (Leica DM2000; Leica Microsystems GmbH). All data were analyzed with Image-Pro plus 6.0 (Media Cybernetics, Inc., Rockville, MD, USA).

Reverse transcription-polymerase chain reaction (RT-PCR). All tissue preparation steps for the isolation of mRNA was performed as previously described (25). The samples not subject to immunohistochemistry were used ( $n=4$ rats per group). Primer pairs for GAPDH and ARNT2 were designed based on sequences in the PubMed database (https://www.ncbi.nlm.nih. gov/pubmed/). The primers for ARNT2 were forward, 5'-ACC AGCGAGACGGGCTGTCA-3' and reverse, 5'-GTGCCCGGC AGGGAATGGAC-3', and for GAPDH were forward, 5'-GGG CTCTCTGCTCCTCCCTCT-3' and reverse, 5'-CCGTTGAAC TTGCCGTGGGT-3'. The cycling parameters for PCR were as follows: An initial cycle (pre-denaturation step) at $94^{\circ} \mathrm{C}$ for $3 \mathrm{~min}$, followed by 35 cycles at $94^{\circ} \mathrm{C}$ for $15 \mathrm{sec}, 68^{\circ} \mathrm{C}$ for $30 \mathrm{sec}$ and $72^{\circ} \mathrm{C}$ for $30 \mathrm{sec}$ and $72^{\circ} \mathrm{C}$ for $10 \mathrm{~min}$. Band intensities were measured and analyzed using an GAS7001B gel image analysis software (UVIband 10.02; UVItec, Cambridge, UK). The expression of target mRNA was measured based on its quantity relative to that of GADPH (relative quality=target gene optical density/GAPDH optical density).

Statistical analysis. Data were presented as the mean \pm standard error of the mean and were analyzed using SPSS 13.0 software (SPSS Inc., Chicago, IL, USA). All data were analyzed by one-way analysis of variance (ANOVA) followed by Fisher's least significant difference post hoc tests. Pearson's correlation analysis was performed to analyse the relationship between target gene expression and behavioral performance. A two-tailed P-value of $<0.05$ was considered to indicate statistical significance.

\section{Results}

Behavioral assessment $B W$. Rats in the MCAO and MCAO+CUMS groups gained less weight than controls at 7 and 28 days after surgery $(\mathrm{P}<0.01)$. After 28 days of the CUMS regimen, rats in the CUMS group exhibited significantly lower BW compared with the controls $(\mathrm{P}<0.01)$, while rats in the MCAO+CUMS group gained significantly less weight compared with those in the MCAO group $(\mathrm{P}<0.01$; Table I).

Sucrose preference. On day 7 after surgery, sucrose preference in the MCAO and MCAO+CUMS groups was decreased compared with in the control group, albeit non-significantly ( $>0.05)$; likewise the sucrose preference index of the CUMS group did not differ significantly compared with the control group ( $\mathrm{P}>0.05)$. After 28 days of the CUMS regimen, the CUMS and MCAO+CUMS groups exhibited significantly decreased sucrose preference indexes compared with the control group $(\mathrm{P}<0.01)$, although no significant difference was determined between the MCAO and control groups ( $\mathrm{P}>0.05)$. Meanwhile, the CUMS and MCAO+CUMS groups displayed significantly decreased sucrose preference indexes compared with the MCAO group $(\mathrm{P}<0.01$; Table II).

OFT. The OFT assessed horizontal motion distance traveled and number of rearings of each rat. On day 7, rats in the MCAO and MCAO+CUMS groups showed significantly less horizontal movement compared with controls $(\mathrm{P}<0.01)$, although rats in the CUMS group did not ( $\mathrm{P}>0.05$; Table III). Similarly, rats in the MCAO and $\mathrm{MCAO}+\mathrm{CUMS}$ groups performed a significanlty lower number of rearings compared with the control group $(\mathrm{P}<0.01)$, while rats in the CUMS group exhibited no significant difference compared with the controls $(\mathrm{P}>0.05$; Table IV). After 4 weeks of the CUMS regiment, rats in the CUMS and MCAO+CUMS groups exhibited significantly less horizontal movement $(\mathrm{P}<0.05)$ and a lower number of rearings $(\mathrm{P}<0.01)$ compared with controls (Tables III and IV).

ARNT2-positive staining. ANOVA demonstrated that the mean optical density of ARNT2-positive neurons in the CA1 and CA3 hippocampal areas of MCAO and MCAO+CUMS rats was significantly higher than in the control group $(\mathrm{P}<0.05$; Fig. 1A,C and D). Although staining in the CUMS group revealed more ARNT2-positive neurons in CA1/3 than controls, the difference was not significant $(\mathrm{P}>0.05$; Fig. 1A,C and D). Additionally, rats in the CUMS group exhibited increased ARNT2 staining in the CA2 area compared with controls $(\mathrm{P}<0.05$; Fig. $1 \mathrm{~B}$ and $\mathrm{D})$; and decreased ARNT2 staining in $\mathrm{CA} 2$ compared with $\mathrm{MCAO}+\mathrm{CUMS}$ rats $(\mathrm{P}<0.01$; Fig. 1B and D).

PCR of ARNT2 mRNA. ARNT2 mRNA levels in the hippocampi of MCAO and MCAO+CUMS rats were significantly increased compared with in controls $(\mathrm{P}<0.05$ and $<0.01$, respectively); while there was no significant difference between the MCAO and MCAO+CUMS groups $(\mathrm{P}>0.05)$. Additionally, ARNT2 mRNA expression was higher 
Table I. Results of weight changes in rats.

BW, g

Postoperative day

\begin{tabular}{lccc} 
Group & Baseline & 7 & 28 \\
\hline Control & $278.38 \pm 9.89$ & $299.19 \pm 9.15$ & $360.69 \pm 16.39$ \\
MCAO & $277.50 \pm 21.93$ & $194.31 \pm 27.13^{\mathrm{a}}$ & $295.44 \pm 41.45^{\mathrm{a}}$ \\
CUMS & $273.00 \pm 21.66$ & $279.75 \pm 16.66$ & $273.25 \pm 20.98^{\mathrm{a}}$ \\
MCAO+CUMS & $279.75 \pm 31.57$ & $208.31 \pm 21.50^{\mathrm{a}}$ & $249.56 \pm 38.44^{\mathrm{a}, \mathrm{b}}$ \\
F-value & 0.134 & 55.126 & 18.718 \\
P-value (ANOVA) & 0.939 & $<0.001$ & $<0.001$
\end{tabular}

BW prior to enrolment, and 7 and 28 days after surgery in the control, MCAO, CUMS and MCAO+CUMS groups (n=8/group). Data are presented as means \pm standard error of the mean. ${ }^{a} \mathrm{P}<0.01$ vs. the control group; ${ }^{b} \mathrm{P}<0.01 \mathrm{vs}$. the $\mathrm{MCAO}$ group. $\mathrm{BW}$, body weight; $\mathrm{MCAO}$, middle cerebral artery occlusion; CUMS, chronic unpredictable mild stress; ANOVA, analysis of variance.

Table II. Sucrose preference index.

Sucrose preference index

\begin{tabular}{lccc}
\hline & & Sucrose preference index \\
\cline { 2 - 4 } Group & Baseline & Postoperative day \\
\cline { 3 - 4 } Control & $0.854 \pm 0.023$ & 7 & 28 \\
MCAO & $0.890 \pm 0.014$ & $0.735 \pm 0.010$ & $0.668 \pm 0.014$ \\
CUMS & $0.873 \pm 0.011$ & $0.708 \pm 0.017$ & $0.633 \pm 0.007$ \\
MCAO+CUMS & $0.874 \pm 0.020$ & $0.710 \pm 0.020$ & $0.457 \pm 0.015^{\mathrm{a}, \mathrm{b}}$ \\
F-value & 0.660 & $0.698 \pm 0.025$ & $0.406 \pm 0.025^{\mathrm{a}, \mathrm{b}}$ \\
P-value (ANOVA) & 0.584 & 0.683 & 60.314 \\
\hline
\end{tabular}

Sucrose preference index prior to enrolment and 7 and 28 days after surgery in the MCAO, CUMS and MCAO+CUMS groups ( $\mathrm{n}=8 / \mathrm{group}$ ). Data are presented as means \pm standard error of the mean. ${ }^{\mathrm{a}} \mathrm{P}<0.01 \mathrm{vs}$. the control group; ${ }^{\mathrm{b}} \mathrm{P}<0.01 \mathrm{vs}$. the MCAO group. MCAO, middle cerebral artery occlusion; CUMS, chronic unpredictable mild stress; ANOVA, analysis of variance.

Table III. Horizontal movement in the OFT.

Movement, $\mathrm{cm} / 5 \mathrm{~min}$

\begin{tabular}{ccc}
\hline & \multicolumn{2}{c}{ Postoperative day } \\
\cline { 2 - 3 } Baseline & 7 & 28 \\
$10,526.62 \pm 746.72$ & $10,798.12 \pm 1,155.86$ & $10,674.85 \pm 1,914.86$ \\
$9,855.28 \pm 2,037.12$ & $2,578.43 \pm 718.61^{\mathrm{a}}$ & $10,980.34 \pm 939.91$ \\
$10,621.12 \pm 2,139.89$ & $10,543.91 \pm 1,975.83$ & $6,630.28 \pm 4,449.65^{\mathrm{b}}$ \\
$10,432.58 \pm 1,332.78$ & $2,504.37 \pm 1,220.60^{\mathrm{a}}$ & $6,385.03 \pm 3,738.11^{\mathrm{b}}$ \\
0.343 & 97.340 & 5.216 \\
0.794 & $<0.001$ & 0.005
\end{tabular}

Horizontal movement in the OFT prior to enrolment and 7 and 28 days after surgery in the control, MCAO, CUMS and MCAO+CUMS groups ( $\mathrm{n}=8$ /group). Data are presented as means \pm standard error of the mean. ${ }^{\mathrm{a}} \mathrm{P}<0.01,{ }^{\mathrm{b}} \mathrm{P}<0.05$ vs. the control group. OFT, open field test; MCAO, middle cerebral artery occlusion; CUMS, chronic unpredictable mild stress; ANOVA, analysis of variance. 
Table IV. Number of rearings in the OFT.

No. of rearings/5 $\mathrm{min}$

\begin{tabular}{lccc}
\cline { 3 - 4 } Groups & & \multicolumn{2}{c}{ Postoperative day } \\
\cline { 3 - 4 } Control & Baseline & 7 & 28 \\
MCAO & $20.00 \pm 4.57$ & $20.50 \pm 2.33$ & $20.38 \pm 8.03$ \\
CUMS & $20.75 \pm 5.04$ & $7.50 \pm 4.21^{\mathrm{a}}$ & $20.50 \pm 3.85$ \\
MCAO+CUMS & $20.25 \pm 6.67$ & $21.00 \pm 3.70$ & $10.13 \pm 10.23^{\mathrm{a}}$ \\
F-value & $19.63 \pm 2.39$ & $7.13 \pm 5.77^{\mathrm{a}}$ & $8.00 \pm 4.87^{\mathrm{a}}$ \\
P-value (ANOVA) & 0.074 & 27.496 & 6.757 \\
\hline
\end{tabular}

Number of rearings in the OFT prior to enrollment and 7 and 28 days after surgery in the control, MCAO, CUMS and MCAO+CUMS groups ( $\mathrm{n}=8$ /group). Data are presented as means \pm standard error of the mean. ${ }^{a} \mathrm{P}<0.01$ vs. the control group. OFT, open field test; MCAO, middle cerebral artery occlusion; CUMS, chronic unpredictable mild stress; ANOVA, analysis of variance.

Table V. Correlation (r-values) between rat behavioral traits and ARNT mRNA expression.

\begin{tabular}{lcccc}
\hline & Body weight & Sucrose preference index & Horizontal movement & Number of rearings \\
\hline ARNT mRNA expression & 0.067 & $0.547^{\mathrm{a}}$ & $0.485^{\mathrm{a}}$ & 0.014 \\
\hline
\end{tabular}

${ }^{\mathrm{a}} \mathrm{P}<0.01$

in the hippocampi of rats in the MCAO+CUMS group compared with in CUMS rats, albeit non-significantly (P>0.05; Fig. 2).

Partial correlation analysis results. The sucrose preference index and horizontal movement distance in the OFT were positively correlated with ARNT mRNA level $(\mathrm{r}=0.547$ and 0.485 ; $\mathrm{P}<0.01)$. ARNT mRNA expression was also positively correlated with body weight $(\mathrm{r}=0.067)$ and number of rearings $(r=0.014)$, though this was not significant $(P>0.05$; Table $V)$.

\section{Discussion}

The results of the current study illustrated that ARNT2 expression in the hippocampus differed among rats following control, CUMS, MCAO and MCAO+CUMS treatments, which is consistent with a previous study describing the effect of transcription factors from the bHLH super-family on post-stroke depression (25). The increased expression of ARNT2 detected in the hippocampi of rats has previously been confirmed to be associated with the vulnerability of distinct dopaminergic projections to hypoxia $(16,31)$, but to our knowledge, the increased expression in the $\mathrm{MCAO}+\mathrm{CUMS}$ group, representing a model of PSD, is a novel finding. Additionally, the observation that there was a trend toward increased ARNT2 levels in the MCAO+CUMS group compared with in the MCAO group is also novel. These alterations in ARNT2 expression were also confirmed at the protein level, which is consistent with a previous study that found HIF- $1 \alpha$-mediated increases in ARNT2 protein expression in neuronal PC12 cells (32). By contrast, another study detected decreased ARNT2 expression in response to HIF-1 $\alpha$ signaling in oral squamous cell carcinoma-derived cell lines (33), which may be attributed to variable cell types and altered cell cycle progression. Although these conflicting findings warrant further research, the focus of the current study was primarily on the change of ARNT2 in a PSD model, and its specific mechanism is still unclear, worthy for further study.

A notable finding was that the hippocampi isolated from the MCAO and MCAO+CUMS groups exhibited increased levels of ARNT2 protein expression as well as mRNA expression, reflecting its potential protective role in resisting stress and promoting neuron survival. Ischemic brain damage leads to secondary ischemic brain tissue hypoxia, brain edema, degeneration, tissue softening, necrosis and inflammation, which in turn induce apoptosis and cell death (34).

Behavioral tests were also utilized to investigate the association between the expression of ARNT2 and behavioral performance. On day 7 after surgery, rats that underwent MCAO gained less weight, consumed slightly less sucrose solution and showed reduced activity, albeit temporarily, as a result of the procedure. After 28 days, rats with MCAO appeared to somewhat recover, although the BW of MCAO rats remained significantly lower compared with that of control rats, potentially as a result of a systemic response to the surgical injury. Moreover, the BW and sucrose consumption of rats subjected to the CUMS regiment were significantly decreased compared 

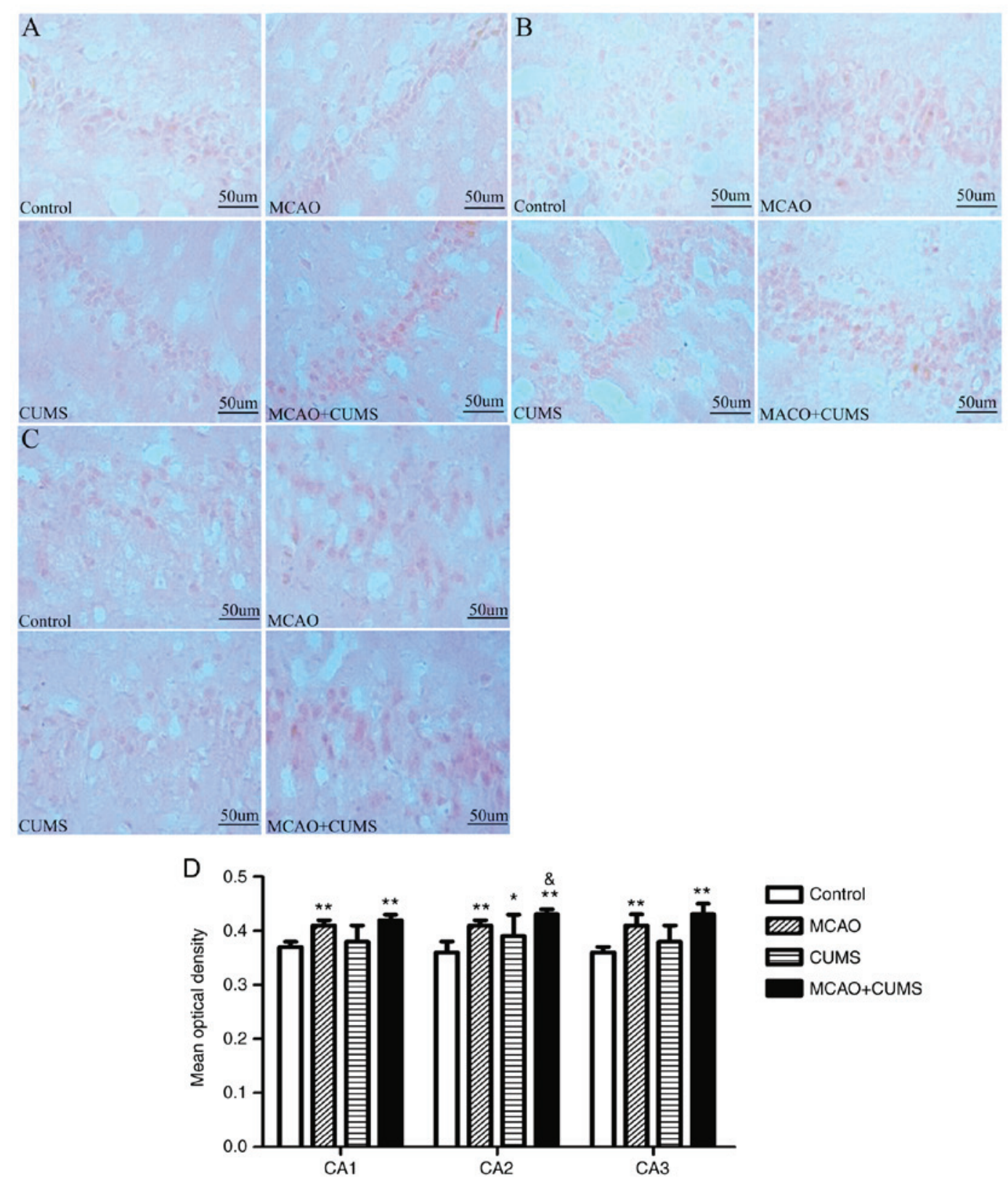

Figure 1. (A-C) Immunohistochemical results of ARNT2-positive staining and (D) mean optical density of staining after 4 weeks in the control, MCAO, CUMS and MCAO+CUMS groups ( $\mathrm{n}=4$ /group). The hippocampal subfields CA1, CA2 and CA3 were observed at x200 magnification. (A-C) show CA1, CA2 and CA3, respectively. " $\mathrm{P}<0.05,{ }^{* *} \mathrm{P}<0.01$ vs. the control group; ${ }^{\circledR} \mathrm{P}<0.01$ vs. the CUMS group. ARNT2, aryl hydrocarbon receptor nuclear translocator protein 2; MCAO, middle cerebral artery occlusion; CUMS, chronic unpredictable mild stress.

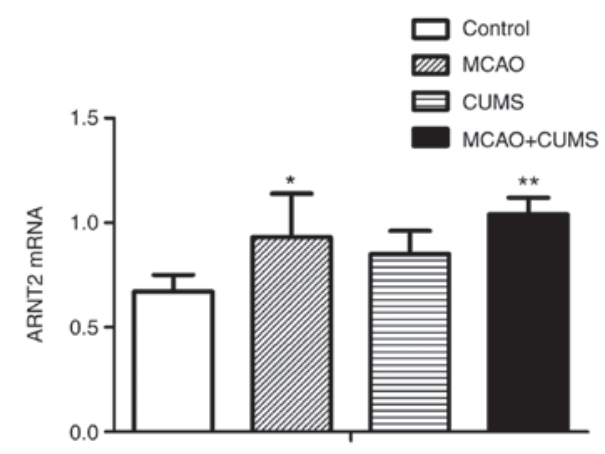

Figure 2. Expression of ARNT2 mRNA relative to GAPDH in the hippocampi of rats after 4 weeks in the control, MCAO, CUMS and MCAO+CUMS groups ( $\mathrm{n}=4 /$ group). ${ }^{*} \mathrm{P}<0.05,{ }^{* *} \mathrm{P}<0.01$ vs. the control group. ARNT2, aryl hydrocarbon receptor nuclear translocator protein 2; MCAO, middle cerebral artery occlusion; CUMS, chronic unpredictable mild stress. with in controls; further, MCAO+CUMS rats gained less weight and consumed less sucrose solution compared with MCAO rats. Collectively, these results indicate successful establishment of the depression and PSD models.

OFT was performed to measure spontaneous activity and independent exploratory behavior, which has previously been used to evaluate depressive behavior (35). In the current study, CUMS and MCAO+CUMS rats subjected to a 4 week-CUMS regiment moved shorter distances and performed a lower number of rearings compared with controls. Furthermore, MCAO+CUMS rats moved shorter distances and performed less rearings than those of the CUMS group, although these differences were not significant. Overall these results indicate that cerebral ischemia together with stress may serve a role in behavioral performance. 
Interestingly, ARNT2 was significantly elevated in the $\mathrm{MCAO}$ and $\mathrm{MCAO}+\mathrm{CUMS}$ groups within penumbra regions of the hippocampus, and therefore may be part of a protective response to this type of injury. Moreover, levels of ARNT were increased, which was previously observed in white blood cells of patients with depression (36). Expectedly, MCAO+CUMS rats also exhibited higher levels of ARNT2 compared with MCAO and CUMS rats. However, the behavioral performance of MCAO+CUMS rats was significantly worse compared with the other groups. Several factors may account for this. First, it may be that the protective role of ARNT2 is limited to compensation for damaged tissue, via upregulation in the hippocampi; however, the regulation underlying this ARNT2 expression has yet to be fully elucidated. Second, the HIF-1 $\alpha$-dependent feed-forward loop that promotes the upregulation of ARNT2, thus inhibiting apoptosis and necrosis of neurons (31), may be prolonged in disease states in order to ensure the survival of neurons in the brain. Maltepe et al (15) demonstrated that ARNT2/HIF-1 $\alpha$ complexes associated with p53 and B-cell lymphoma 2 family members to modulate apoptosis in response to hypoxia/ischemia in neurons and other tissues. It has also been indicated that chronic stress has a negative impact on neurogenesis, with a previous study showing that cell proliferation and survival, as well as neuronal differentiation, declined in stressed rodents (25). Therefore, it may be worthwhile investigating whether and how the survival and apoptosis of neurons in the prefrontal cortex and hippocampus are affected in PSD.

Previously, our group demonstrated that the bHLH-PAS factor, NPAS4, which dimerizes with ARNT2 and subsequently binds to brain-derived neurotrophic factor promoter-I to modulate nerve-excitability-dependent transcription, was significantly decreased in CUMS and MCAO+CUMS rats (25). Therefore, it is possible that ARNT2 is involved in mechanisms underlying gene-environment interactions in psychiatric models, but the specific biological signaling pathways require further investigation. Additionally, further research is warranted to clarify HIF-1 activity in neurons, using ARNT2-deficient rats or ARNT2 antagonists/agonists. Nevertheless, the results of the present study regarding the expression of ARNT2 in the hippocampus may serve as a basis for further study into the etiology of psychiatric disorders following cerebral ischemia.

\section{Acknowledgements}

Not applicable.

\section{Funding}

The current study was supported by the National Natural Science Foundation of China (grant no. 81471349), Henan Science and Technology Development Project (grant no. 162102310489), the Natural Science Foundation of Henan Province (grant no. 162300410224), Research on the Open Group of Phychiatric Medicine in Xinxiang Medical College (grant no. 2016PN-KFKT-09), the Training Plan for Young Excellent Teachers in Colleges and Universities of Henan (grant no. 2016GGJS-106) and the Science and Technology
Project of Xinxiang (grant no. CXGG17030), and additional support was provided by the 2016 Graduate Scientific Research Innovation Support Program of Xinxiang (grant no. YJSCX201625Y).

\section{Availability of data and materials}

All data generated or analyzed during this study are included in this published article.

\section{Authors' contributions}

WW made substantial contributions to conception and design of the study. LZ performed the experiments on animal behavior. SG was responsible for the polymerase chain reaction and immunohistochemistry experiments. XW collected the data. PF analyzed and interpreted data. CP drafted the manuscript. XZ revised the manuscript critically for important intellectual content. WL and JM ensured that questions related to the accuracy or integrity of any part of the work were appropriately investigated and resolved. ZZ and JS gave final approval of the version to be published.

\section{Ethics approval and consent to participate}

All experiments involving animals were approved by the Laboratory Animals Management Commission of the Henan Key Laboratory of Biological Psychiatry, Xinxiang, China.

\section{Patient consent for publication}

Not applicable.

\section{Competing interests}

The authors declare that they have no competing interests.

\section{References}

1. Kronenberg G, Gertz K, Heinz A and Endres M: Of mice and men: Modelling post-stroke depression experimentally. Br J Pharmacol 171: 4673-4689, 2014.

2. Robinson RG and Jorge RE: Post stroke depression: A review. Am J Psychiatry 173: 221-231, 2016.

3. Nabavi SF, Habtemariam S, Di Lorenzo A, Sureda A, Khanjani S, Nabavi SM and Daglia M: Post-stroke depression modulation and in vivo antioxidant activity of gallic acid and its synthetic derivatives in a murine model system. Nutrients 8: E248, 2016.

4. Mozaffarian D, Benjamin EJ, Go AS, Arnett DK, Blaha MJ, Cushman M, de Ferranti S, Després JP, Fullerton HJ, Howard VJ, et al: Heart disease and stroke statistics-2015 update: A report from the American Heart Association. Circulation 131: e29-e322, 2015.

5. Wang S, Sun H, Liu S, Wang T, Guan J and Jia J: Role of hypothalamic cannabinoid receptors in post-stroke depression in rats. Brain Res Bull 121: 91-97, 2016.

6. Swartz RH, Bayley M, Lanctôt KL, Murray BJ, Cayley ML, Lien K, Sicard MN, Thorpe KE, Dowlatshahi D, Mandzia JL, et al: Post-stroke depression, obstructive sleep apnea, and cognitive impairment: Rationale for, and barriers to, routine screening. Int J Stroke 11: 509-518, 2016.

7. Nabavi SF, Dean OM, Turner A, Sureda A, Daglia M and Nabavi SM: Oxidative stress and post stroke depression: Possible therapeutic role of polyphenols? Curr Med Chem 22: 343-351, 2015.

8. Loubinoux I, Kronenberg G, Endres M, Schumann-Bard P, Freret T, Filipkowski RK, Kaczmarek L and Popa-Wagner A: Post-stroke depression: Mechanisms, translation and therapy. J Cell Mol Med 16: 1961-1969, 2012. 
9. Nabavi SF, Turner A, Dean O, Sureda A and Mohammad S: Post stroke depression therapy: Where are we now? Curr Neurovasc Res 11: 279-289, 2014.

10. Drutel G, Héron A, Kathmann M, Gros C, Macé S, Plotkine M, Schwartz JC and Arrang JM: ARNT2 a transcription factor for brain neuron survival? Eur J Neurosci 11: 1545-1553, 1999.

11. Dougherty EJ and Pollenz RS: Analysis of Ah receptor-ARNT and Ah receptor-ARNT2 complexes in vitro and in cell culture. Toxicol Sci 103: 191-206, 2008.

12. Rajashekaran P, Pai K, Thunga R and Unnikrishnan B: Post-stroke depression and lesion location: A hospital based cross-sectional study. Indian J Psychiatry 55: 343-348, 2013.

13. Keith B, Adelman DM and Simon MC: Targeted mutation of the murine arylhydrocarbon receptor nuclear translocator 2 (Arnt2) gene reveals partial redundancy with Arnt. Proc Natl Acad Sci USA 98: 6692-6697, 2001.

14. Corcoran SE and O'Neill LA: HIF1 $\alpha$ and metabolic reprogramming in inflammation. J Clin Invest 126: 3699-3707, 2016.

15. Maltepe E, Keith B, Arsham AM, Brorson JR and Simon MC: The role of ARNT2 in tumor angiogenesis and the neural response to hypoxia. Biochem Biophys Res Commun 273: 231-238, 2000.

16. Dela Cruz JA, Schmidt-Kastner R, Stevens JA, Steinbusch HW and Rutten BP: Differential distribution of hypoxia-inducible factor 1-beta (ARNT or ARNT2) in mouse substantia nigra and ventral tegmental area. J Chem Neuroanat 61-62: 64-71, 2014.

17. den Heijer $\mathrm{T}$, Tiemeier $\mathrm{H}$, Luijendijk HJ, van der Lijn F, Koudstaal PJ, Hofman A and Breteler MM: A study of the bidirectional association between hippocampal volume on magnetic resonance imaging and depression in the elderly. Biol Psychiatry 70: 191-197, 2011.

18. Kempton MJ, Salvador Z, Munafò MR, Geddes JR, Simmons A, Frangou S and Williams SC: Structural neuroimaging studies in major depressive disorder. Meta-analysis and comparison with bipolar disorder. Arch Gen Psychiatry 68: 675-690, 2011.

19. Erickson KI, Prakash RS, Voss MW, Chaddock L, Heo S, McLaren M, Pence BD, Martin SA, Vieira VJ, Woods JA, et al: Brain-derived neurotrophic factor is associated with age-related decline in hippocampal volume. J Neurosci 30: 5368-5375, 2010.

20. Li G, Peskind ER, Millard SP, Chi P, Sokal I, Yu CE, Bekris LM, Raskind MA, Galasko DR and Montine TJ: Cerebrospinal fluid concentration of brain-derived neurotrophic factor and cognitive function in non-demented subjects. PLoS One 4: e5424, 2009.

21. Zhang ZH, Wu LN, Song JG and Li WQ: Correlations between cognitive impairment and brain-derived neurotrophic factor expression in the hippocampus of post-stroke depression rats. Mol Med Rep 6: 889-893, 2012.

22. Zhao DA, Bi LY, Huang Q, Zhang FM and Han ZM: Isoflurane provides neuroprotection in neonatal hypoxic ischemic brain injury by suppressing apoptosis. Braz J Anesthesiol 66: 613-621, 2016.

23. Xu Y, Tian Y, Tian Y, Li X and Zhao P: Autophagy activation involved in hypoxic-ischemic brain injury induces cognitive and memory impairment in neonatal rats. J Neurochem 139: 795-805, 2016.

24. Valen E, Pascarella G, Chalk A, Maeda N, Kojima M, Kawazu C, Murata M, Nishiyori H, Lazarevic D, Motti D, et al: Genome-wide detection and analysis of hippocampus core promoters using DeepCAGE. Genome Res 19: 255-265, 2008.
25. Zhang Z, Fei P, Mu J, Wang H, Li W and Song J: Decreased expression of neuronal Per-Arnt-Sim domain protein 4 gene in the hippocampus of a post-stroke depression rat model. Exp Ther Med 7: 1045-1049, 2014

26. Willner P: Chronic mild stress (CMS) revisited: Consistency and behavioural-neurobiological concordance in the effects of CMS. Neuropsychobiology 52: 90-110, 2005.

27. Chen Q, Ren L, Min S, Hao X, Chen H and Deng J: Changes in synaptic plasticity are associated with electroconvulsive shock-induced learning and memory impairment in rats with depression-like behavior. Neuropsychiatr Dis Treat 14: 1737-1746, 2018.

28. Ren L, Hao X, Min S, Deng J, Chen Q, Chen H and Liu D: Anesthetics alleviate learning and memory impairment induced by electroconvulsive shock by regulation of NMDA receptor-mediated metaplasticity in depressive rats. Neurobiol Learn Mem 155: 65-77, 2018.

29. Araujo SM, Poetini MR, Bortolotto VC, de Freitas Couto S, Pinheiro FC, Meichtry LB, de Almeida FP, Santos Musachio EA, de Paula MT and Prigol M: Chronic unpredictable mild stress-induced depressive-like behavior and dysregulation of brain levels of biogenic amines in Drosophila melanogaster. Behav Brain Res 351: 104-113, 2018.

30. Shao QY, You F, Zhang YH, Hu LL, Liu WJ, Liu Y, Li J, Wang SD and Song MF: CSF miR-16 expression and its association with miR-16 and serotonin transporter in the raphe of a rat model of depression. J Affect Disord 238: 609-614, 2018.

31. Mandl M and Depping R: Hypoxia-inducible aryl hydrocarbon receptor nuclear translocator (ARNT)(HIF-1 $\beta$ ): Is it a rare exception? Mol Med 20: 215-220, 2014.

32. Mandl M, Lieberum MK and Depping R: A HIF-1 $\alpha$-driven feed-forward loop augments HIF signalling in Hep3B cells by upregulation of ARNT. Cell Death Dis 7: e2284, 2016

33. Kimura Y, Kasamatsu A, Nakashima D, Yamatoji M, Minakawa Y, Koike K, Fushimi K, Higo M, Endo-Sakamoto Y, Shiiba M, et al: ARNT2 regulates tumoral growth in oral squamous cell carcinoma. J Cancer 7: 702-710, 2016.

34. Arteaga O, Álvarez A, Revuelta M, Santaolalla F, Urtasun A and Hilario E: Role of antioxidants in neonatal hypoxic-ischemic brain injury: New therapeutic approaches. Int J Mol Sci 18: E265, 2017.

35. Kim YR, Kim HN, Pak ME, Ahn SM, Hong KH, Shin HK and Choi BT: Studies on the animal model of post-stroke depression and application of antipsychotic aripiprazole. Behav Brain Res 287: 294-303, 2015.

36. Kajta M, Wnuk A, Rzemieniec J, Litwa E, Lason W, Zelek-Molik A, Nalepa I, Rogóż Z, Grochowalski A and Wojtowicz AK: Depressive-like effect of prenatal exposure to DDT involves global DNA hypomethylation and impairment of GPER1/ESR1 protein levels but not ESR2 and AHR/ARNT signaling. J Steroid Biochem Mol Biol 171: 94-109, 2017.

This work is licensed under a Creative Commons Attribution-NonCommercial-NoDerivatives 4.0 International (CC BY-NC-ND 4.0) License. 